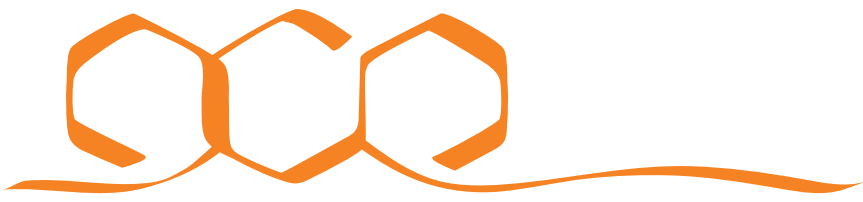 \\ COMMUNICATIONS \\ CHEMISTRY
}

ARTICLE

https://doi.org/10.1038/s42004-020-00442-6 OPEN

\section{Role of hydroxylation for the atomic structure of a non-polar vicinal zinc oxide}

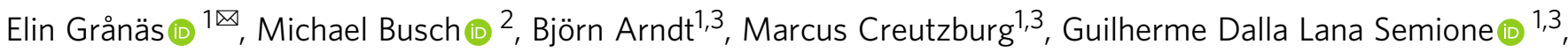 \\ Johan Gustafson (1) 4, Andreas Schaefer ${ }^{4,5}$, Vedran Vonk (1) 1, Henrik Grönbeck ${ }^{2}$ \& Andreas Stierle (1) 1,3凶
}

From the catalytic, semiconducting, and optical properties of zinc oxide $(\mathrm{ZnO})$ numerous potential applications emerge. For the physical and chemical properties of the surface, undercoordinated atoms often play an important role, necessitating systematic studies of their influence. Here we study the vicinal $\mathrm{ZnO}(10 \overline{1} 4)$ surface, rich in under-coordinated sites, using a combination of several experimental techniques and density functional theory calculations. We determine the atomic-scale structure and find the surface to be a stable, long-range ordered, non-polar facet of $\mathrm{ZnO}$, with a high step-density and uniform termination. Contrary to an earlier suggested nano-faceting model, a bulk termination fits much better to our experimental observations. The surface is further stabilized by dissociatively adsorbed $\mathrm{H}_{2} \mathrm{O}$ on adjacent under-coordinated $\mathrm{O}$ - and $\mathrm{Zn}$-atoms. The stabilized surface remains highly active for water dissociation through the remaining under-coordinated $\mathrm{Zn}$-sites. Such a vicinal oxide surface is a prerequisite for future adsorption studies with atomically controlled local step and terrace geometry.

\footnotetext{
${ }^{1}$ Deutsches Elektronen-Synchrotron (DESY), 22607 Hamburg, Germany. ${ }^{2}$ Department of Physics and Competence Centre for Catalysis, Chalmers University of Technology, 41296 Göteborg, Sweden. ${ }^{3}$ Fachbereich Physik, Universität Hamburg, 20355 Hamburg, Germany. ${ }^{4}$ Division of Synchrotron Radiation Research, Lund University, 22100 Lund, Sweden. ${ }^{5}$ Department of Chemistry and Chemical Engineering and Competence Centre for Catalysis, Chalmers University of Technology, 41296 Göteborg, Sweden. ${ }^{凶}$ email: e.granas@protonmail.com; andreas.stierle@desy.de
} 
Z inc oxide $(\mathrm{ZnO})$ receives much attention owing to its exceptional catalytic, semiconducting, and optical properties $^{1,2}$. In applications involving $\mathrm{ZnO}$ surfaces, under-coordinated sites like steps and edges play an important role $^{3}$. To systematically study the influence of atomic step sites on the adsorption behavior as a function of the step-density or orientation, vicinal surfaces have proven very useful ${ }^{4}$. Such surfaces are composed of terraces of a low-index orientation separated by parallel steps in dense, highly ordered arrays. For metals there are numerous studies applying vicinal surfaces to elucidate the role of step edges in catalysis ${ }^{4-7}$. Vicinal oxides are, however, generally much less studied and are often poorly understood on an atomic-scale. Exceptions are $\mathrm{Al}_{2} \mathrm{O}_{3}{ }^{8-12}$ and $\mathrm{SrTiO}_{3}{ }^{13-17}$ for which several vicinal surfaces and their use as support for lowdimensional nanostructures was explored. Another example is vicinal $\mathrm{TiO}_{2}$, which has also been studied in some detail, providing insights in the stability and structure of steps and their influence on the electronic structure of the surface and in the role of step sites on the interaction with $\mathrm{O}_{2}{ }^{18,19}$. Despite the observation that the presence of surface steps on $\mathrm{ZnO}$ influences the decomposition and reaction products in heterogeneous catalysis ${ }^{20-23}$, a systematic investigation of vicinal $\mathrm{ZnO}$ surfaces correlating the structure and reactivity is lacking.

Owing to alternating layers of $\mathrm{Zn}^{2+}$ and $\mathrm{O}^{2-}$ in the nominal bulk-terminated $\mathrm{ZnO}$ wurtzite structure, many of the surface orientations are polar and thus unstable. The stabilization mechanisms for the polar, low-index $\mathrm{ZnO}(0001)$ and $(000 \overline{1})$ surfaces were studied in detail, showing that the non-zero dipole moment of the surface is canceled by compensating the charge through reconstructions, leading to changes in the surface stoichiometry or by adsorbates, especially $\mathrm{OH}^{24-37}$. It was also proposed that hydrogen in subsurface sites contributes to the stabilization of the surface ${ }^{38,39}$. Hydroxylation at oxide surfaces is a generally observed phenomenon, for polar and non-polar surfaces alike. This raises the question which role hydroxylation has for $\mathrm{ZnO}$. Understanding the effect of hydroxylation is also important for a better understanding of the shape and reactivity of $\mathrm{ZnO}$ nanoparticles. The most stable surface for $\mathrm{Zn}$-terminated $\mathrm{ZnO}$ was suggested to be the (1014) surface ${ }^{40-42}$, a surface with an angle of $24.8^{\circ}$ to the (0001)-surface. For the O-terminated $\mathrm{ZnO}$

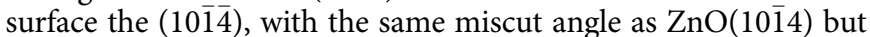
to the $(000 \overline{1})$-surface instead of $(0001)$, is suggested as the most stable ${ }^{43}$. The bulk-terminated $\mathrm{ZnO}(10 \overline{1} 4)$ is an oxide surface of Tasker type $2^{44}$, thus having zero total dipole moment of the surface unit cell. This high step-density vicinal surface thus is potentially stable owing to its lack of macroscopic dipole moment.

Here, we have employed a combination of surface X-ray diffraction (SXRD), scanning tunneling microscopy (STM), X-ray photoelectron spectroscopy (XPS), and density functional theory (DFT) to study the $\mathrm{ZnO}(10 \overline{1} 4)$ surface. We find that this is a very stable $\mathrm{ZnO}$ surface with a potentially high reactivity due to the high density of under-coordinated sites. We identify the $\mathrm{ZnO}$ (1014) surface to be bulk truncated, contrary to the earlier suggested nano-faceting model. Furthermore, our calculations show that $\mathrm{OH}$ groups are present on the surface even under the best ultra-high vacuum conditions at room temperature and additionally stabilize the $\mathrm{ZnO}(10 \overline{1} 4)$ surface. This prediction is supported by a comparison of experimental $\mathrm{O}$ 1s core levels and theoretically calculated core level shifts for the clean $\mathrm{ZnO}(10 \overline{1} 4)$ surface and in the presence of $\mathrm{OH}$ groups.

\section{Results and discussion}

Surface characterization with STM and low-energy electron diffraction (LEED). Figure 1(a) shows a representative STM image of the as prepared $\mathrm{ZnO}(10 \overline{1} 4)$ surface. The surface consists
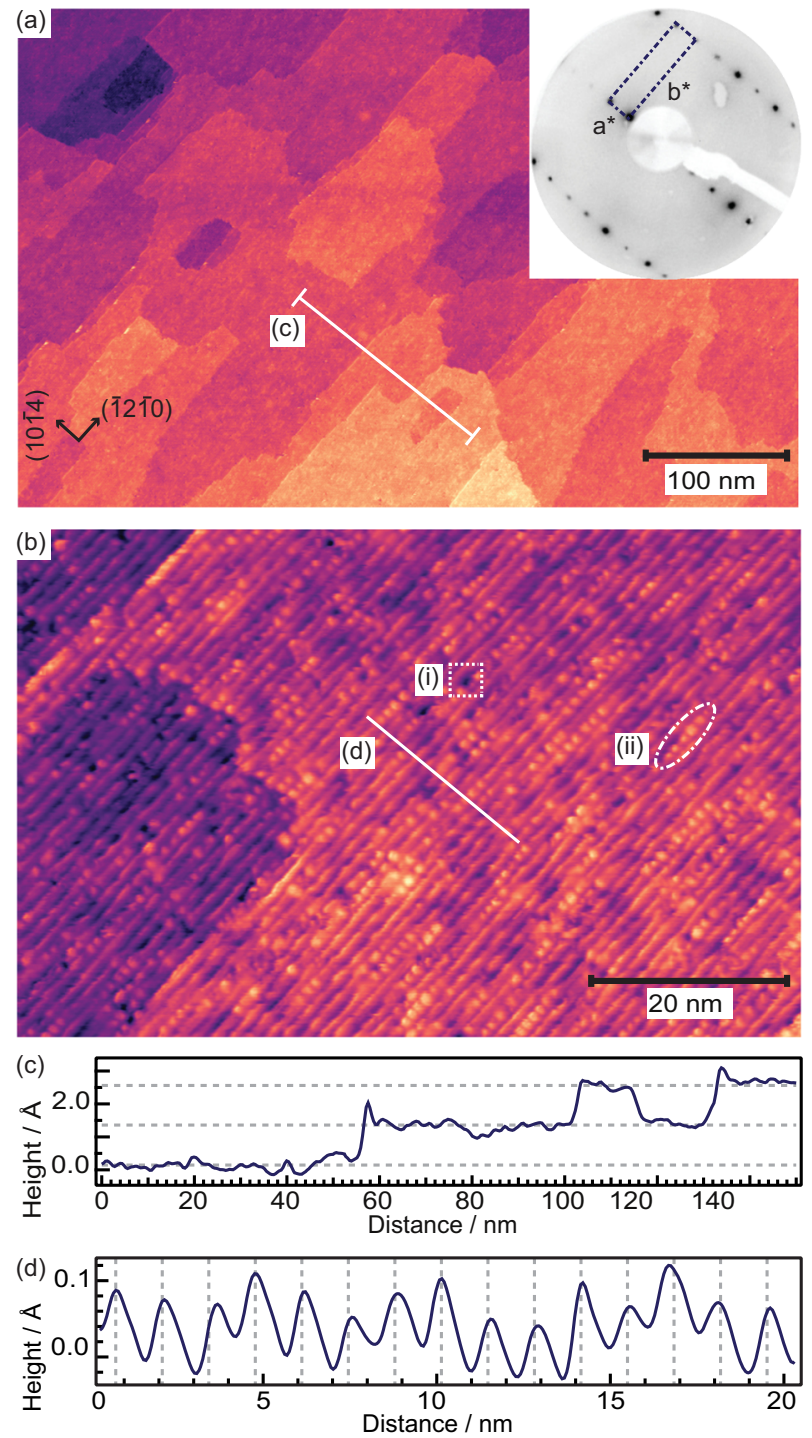

Fig. 1 STM characterization of the ZnO(10ī4) surface. a STM image of surface prepared by annealing for $10 \mathrm{~min}$ at $650^{\circ} \mathrm{C} .550 \times 350 \mathrm{~nm}^{2}, I=0.2$ $\mathrm{nA}, U=1.8 \mathrm{~V}$. Inset: LEED pattern of the $\mathrm{ZnO}(10 \overline{1} 4)$-surface $(52 \mathrm{eV})$ with the surface unit cell marked by the dashed rectangle. b STM image of the as prepared $\mathrm{ZnO}(10 \overline{1} 4)$ surface. $80 \times 50 \mathrm{~nm}^{2}, I=0.2 \mathrm{nA}, U=1.8 \mathrm{~V}$. Dotted squares: type (i) defects, dash-dotted ovals: type (ii) defects. c Line scan across several terraces along the line indicated (a). Averaged over $20 \mathrm{~nm}$. Dashed lines indicate the determined average height between terraces: $1.2 \AA$. $\mathbf{d}$ Line scan perpendicular to the steps along the line indicated in $\mathbf{b}$ Dashed lines indicate the average step periodicity of $13.1 \AA$.

of large terraces (avg. size $1080 \mathrm{~nm}^{2}$ ) elongated in the step edge direction. The average surface roughness is $1.4 \AA$. As comparison, this is in between the previously observed roughness of $<1 \AA$ for the (0001), (0001), and (1010), and $2.5 \AA$ for the (112̄0) surface ${ }^{24}$. The steps of the $\mathrm{ZnO}(10 \overline{1} 4)$ surface appear as stripes, better seen in the smaller STM image in panel (b). Analyzing a large number of images from different preparations we find the height between terraces to be $1.2 \pm 0.1 \AA$ and the step periodicity $13.1 \pm 2.4 \AA$. The terrace height can be seen in the line scan in panel (c) [taken along the line in panel (a)]. The dashed lines indicate the determined height between terraces. The separation between the terraces is identical to the theoretical value of the distance between lattice planes for the $\mathrm{ZnO}(10 \overline{1} 4)$ surface, $1.18 \AA$, in line with a uniform surface termination. In Fig. 1(d) a line scan taken perpendicular to 
the steps in panel (b) show the step periodicity. The step periodicity is in good agreement with the theoretical value $a=12.4 \AA$ ( $b=3.29 \AA)$ and with that previously observed by Zheng et al. ${ }^{40}$ on the faceted $\mathrm{ZnO}(0001)$ surface. For vicinal surfaces with periodic arrays of steps the LEED pattern is expected to exhibit a spot splitting around the fundamental signals of the low-index surface, in this case (0001), where the separation between the spots are the reciprocal of the average periodicity of the vicinal surface ${ }^{45}$. The inset in panel (a) shows the LEED pattern of the as prepared $\mathrm{ZnO}$ (1014) surface, where the spot-slitting expected for a nonreconstructed $\mathrm{ZnO}(10 \overline{1} 4)$ surface can clearly be seen. The welldefined spots and the absence of smearing indicate that the surface is both well-ordered on a larger scale and has a narrow distribution of step widths. We have discussed the LEED pattern of the $\mathrm{ZnO}(10 \overline{1} 4)$ surface in more detail in a previous publication. ${ }^{42}$

Two types of defects are also seen in Fig. 1(a): (i) dark points appearing as "dents" on the steps and (ii) bright protrusions along the steps. An example of type (i) is marked by a dotted square and type (ii) by a dash-dotted oval. Though the lack of atomic resolution makes it hard to draw any definite conclusions we attempt to assign the defects as follows. The concentration of type (i) defects does not change with time in vacuum, after exposure to $\mathrm{O}_{2}$ (see Fig. S1 and surrounding discussion), or after repeated scanning in the same area. Based on their inertness we tentatively assign these defects to $\mathrm{Zn}$ - or $\mathrm{ZnO}$ vacancies. This is in line with studies on the (1010) $\mathrm{ZnO}$ surface, where $\mathrm{ZnO}$ vacancies have been shown to be the thermodynamically most favorable type of atomic defect ${ }^{46}$. Type (ii) defects, in contrast, slowly increase in number with time when the sample is kept at room temperature, indicating that they are due to adsorbates. We assign them to $\mathrm{OH}$ accumulating on the surface during the measurement times (up to $10 \mathrm{~h}$ ), despite very good vacuum conditions in the low $10^{-11}$ mbar regime. Adsorption of $\mathrm{OH}$ from the background pressure is in line with our XPS measurements (see Figs. 2 and S2) and DFT calculations, which indicate that partially dissociative water adsorption at the $\mathrm{ZnO}(10 \overline{1} 4)$ surface is strongly exothermic. Similar to our STM observations on the $\mathrm{ZnO}(1014)$ surface, two types of defects were observed with atomic force microscopy and

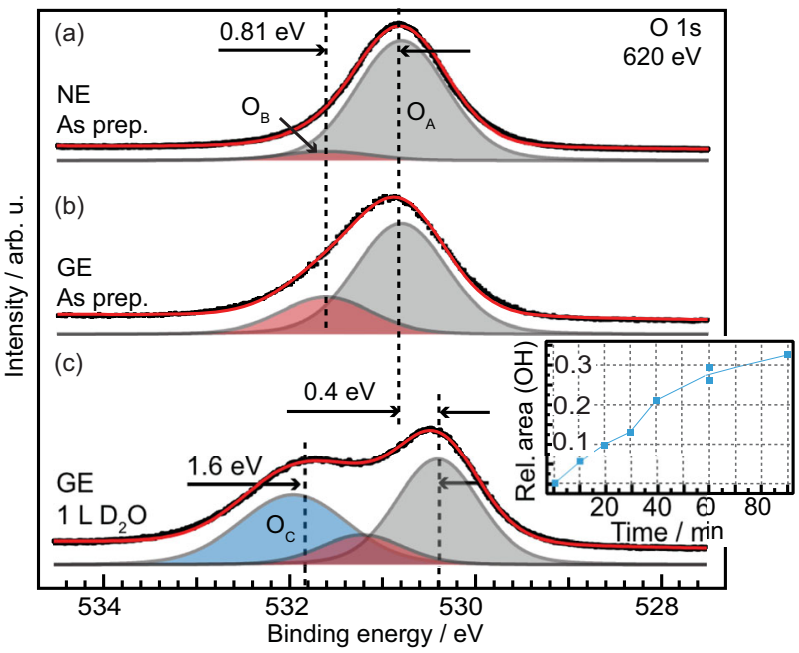

Fig. 2 Measured $\mathbf{O}$ 1s core level spectra. a $\mathrm{O}$ 1s of $\mathrm{ZnO}(10 \overline{1} 4)$ for the as prepared surface measured in normal- and $\mathbf{b}$ grazing emission. $\mathbf{c} \mathrm{O}$ 1s after exposure to $1 \mathrm{~L} \mathrm{D}_{2} \mathrm{O}$ at room temperature, grazing emission. Black dots: measured data. Red line: fitted spectra. Shaded peaks: fitted components as labeled in the figure. Inset: Peak area of the XPS O 1s $\mathrm{O}_{\mathrm{C}}$ component, normalized to the water saturated spectrum, as a function of time in $1 \cdot 10^{-10}$ mbar residual gas, mainly composed of $\mathrm{H}_{2}$ and $\mathrm{H}_{2} \mathrm{O}$. Line to guide the eye. assigned to $\mathrm{Zn}$-vacancies and $\mathrm{H}$ adsorbed on $\mathrm{Zn}$-atoms, respectively, on a reconstructed $\mathrm{Zn}$-terminated $\mathrm{ZnO}(0001)$ surface $^{33}$.

Based purely on our STM measurements we cannot exclude that some of the defects are subsurface hydrogen, as has previously been suggested to occur on $\mathrm{ZnO}^{38,39,47}$ and also observed on hydroxylated $\mathrm{TiO}_{2}{ }^{48}$. However, significant amounts of subsurface $\mathrm{OH}$ can only form from atomic hydrogen ${ }^{47,49}$ and in our case the only source of hydrogen is from dissociated $\mathrm{H}_{2} \mathrm{O}$ and $\mathrm{H}_{2}$ from the residual gases. In a study by Hellström et $\mathrm{al}^{38}$. subsurface $\mathrm{OH}$ was found on a surface not previously exposed to atomic hydrogen; in this study they measured a significant XPS component corresponding to $\mathrm{OH}$ core level shifts (CLS) of $+1.5-2 \mathrm{eV}$ with respect to the oxide component on the freshly cleaned surface. This was attributed to $\mathrm{OH}$ in the second layer, which contribute to the stabilization of the $c(\sqrt{12} \times \sqrt{12}) \mathrm{R} 30^{\circ}$ surface reconstruction. The $\mathrm{OH}$ component observed by Hellström et al. corresponds to our $\mathrm{O}_{C}$ component, and as we do not observe the $\mathrm{O}_{C}$ component on the freshly cleaned surface (see Figs. 2 and S3 and discussion thereof) we exclude significant amounts of subsurface $\mathrm{OH}$ in the near surface region in our case. Subsurface $\mathrm{OH}$ was also suggested in a X-ray standing wave (XSW) study of the hydroxylated $\mathrm{ZnO}(0001)$ surface ${ }^{39}$. Here, they observe a large in-plane disorder of the oxygen and suggest that $\mathrm{OH}$ has substituted oxygen lattice sites in the near surface region, as well as populate sites along the edges of triangular islands on the surface. Our results may be in line with this finding despite our exclusion of subsurface $\mathrm{OH}$. In our final model [see Fig. 3(b)] we observe two $\mathrm{OH}$ species with different in-plane coordinates, such species would appear as in-plane disorder in XSW measurements.

Investigation of the surface chemical composition by XPS. To obtain chemical information of the surface also XPS measurements were performed. The $\mathrm{O}$ 1s spectra of as-grown $\mathrm{ZnO}(10 \overline{1} 4)$ are shown in Fig. 2 measured in (a) normal emission [NE] and (b) grazing emission at $60^{\circ}[\mathrm{GE}]$. The $\mathrm{O}$ 1s XP-spectra for both measurement geometries can be de-convoluted in two components of identical position and width: the main component, $\mathrm{O}_{A}$ at $530.8 \mathrm{eV}$ assigned to $\mathrm{O}^{2-}$ in wurtzite $\mathrm{ZnO}^{30,50-52}$, and a second component, $\mathrm{O}_{B}$, shifted $+0.81 \pm 0.03 \mathrm{eV}$ from the $\mathrm{O}_{A}$ component. The shift of $\mathrm{O}_{B}$ is significantly lower than the 1.4-2.1 eV that was reported for $\mathrm{OH}$ adsorbed on low-index $\mathrm{ZnO}$ surfaces ${ }^{30,50,52,53}$. The relative intensity of the $\mathrm{O}_{B}$ component is increasing when going from NE to GE (from $7 \%$ to $33 \%$ ), indicating that it is attributed to surface oxygen species.

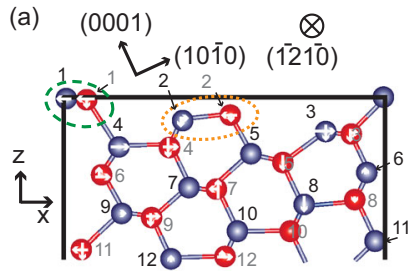

(b)
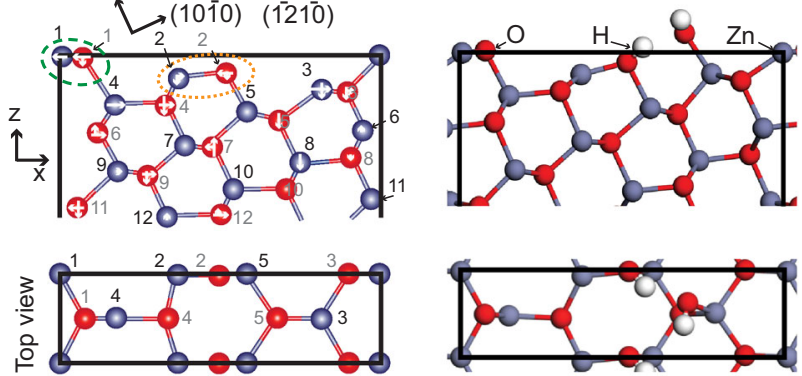

Fig. 3 Structural models of the $\mathrm{ZnO}(\mathbf{1 0} \overline{1} 4)$ unit cell. a The bulk truncated surface model. White arrows indicate the fitted displacements. Atomic colorcode: $\mathrm{H}$ (white), O (red), Zn (blue). Orange dotted oval: Zn-O pair removed for Model B, Green dashed oval: Zn-O pair removed for Model C. Numbers correspond to the labels in Table SI. b DFT optimized structure based on Model $\mathrm{A}$, including one dissociated $\mathrm{H}_{2} \mathrm{O}$ molecule per unit cell. 
The surface was also exposed to $1 \mathrm{~L}_{2} \mathrm{O}$ at room temperature (3.5 $\cdot 10^{-10}$ mbar). The spectrum after exposure is shown in Fig. 2 (c), where a large peak shifted $+1.6 \mathrm{eV}$ from the main peak is seen to appear. This new component, $\mathrm{O}_{C}$, we assign to $\mathrm{OH}$ and $\mathrm{H}_{2} \mathrm{O}$ adsorbed on the surface. We exclude that this peak corresponds to $\mathrm{CO}$ or $\mathrm{CO}_{2}$, see Fig. S3 for the corresponding $\mathrm{C} 1$ s spectra and further discussion. The $\mathrm{O}_{C}$ component is also seen to increase when a freshly prepared surface is exposured to ultra-high vacuum (UHV) for longer times, see inset in Fig. 2(c), through adsorption of water from the background pressure and we correlate this with the type (ii) defects seen in STM (see previous section). The as-prepared surface does not exhibit any $\mathrm{O}_{C}$ component, which would be expected if significant amounts of subsurface $\mathrm{OH}$ was present. The XP-spectra corresponding to the data points in the inset are shown in Fig. S2. After exposure to $\mathrm{D}_{2} \mathrm{O}$ a peak shift of $0.4 \mathrm{eV}$ is observed for the $\mathrm{O}_{A}$ and $\mathrm{O}_{B}$ components in GE. In NE, however (see Fig. S4), the shift is 0.17 $\mathrm{eV}$. All spectra are calibrated to the Fermi-level of a $\mathrm{Cu}(111)$ crystal in electrical contact with the $\mathrm{ZnO}(10 \overline{1} 4)$ crystal. The $\mathrm{Cu}$ Fermi-level can be assumed to not be affected at these experimental conditions, and it can thus be excluded that the shift is an error in binding energy calibration. Instead we assign this peak shift to an adsorption induced metallization in the surface region, with a downward bending of the bands close to the surface. This is exclusively a surface phenomenon and only extends a few nanometers into the bulk ${ }^{54}$, which explains why the observed shift scales with the probing depth (the probing depth for the $\mathrm{O} 1 \mathrm{~s}$ spectra in NE and GE is estimated to be $8.1 \AA$ and $4 \AA$, respectively). Though the surface metallization is more pronounced for $\mathrm{H}$-adsorption it was also observed for $\mathrm{ZnO}$ exposed to water and methanol ${ }^{55}$.

Determination of surface structure using SXRD. To obtain information on the atomic structure of the $\mathrm{ZnO}(10 \overline{1} 4)$ surface, surface X-ray diffraction experiments were performed. A set of crystal truncation rod (CTR) data was obtained from the as prepared surface, no signal from potential super-structures was detected. Three different models were tested against the CTR data and used as starting points for further refinement: A-the bulk truncated surface model seen in Fig. 3(a), B-the model suggested by Zheng et al. ${ }^{40}$ where one under-coordinated $\mathrm{Zn}-\mathrm{O}$ pair is removed from the surface (indicated by the orange dotted oval), $\mathrm{C}-\mathrm{a}$ model where the topmost under-coordinated $\mathrm{Zn}-\mathrm{O}$ pair is removed from the surface (green dashed oval). The DFT relaxed structures of all three models are shown in Fig. S5. All three surfaces exhibit a mixed-terminated stepped structure with (0001)-planes at an angle of $24.8^{\circ}$ to the surface and a periodicity of $12.4 \AA$ perpendicular to the steps. In principle, the three models cannot be differentiated based on coordination; all these surfaces have the same amount of under-coordinated atoms. The positions of $\mathrm{Zn}$ - and $\mathrm{O}$ - atoms in the first seven layers along the surface normal were refined. Owing to the mirror symmetry of the structure, the atoms' $y$ positions were kept fixed and the $x$ and $z$-positions were allowed to vary. These directions are perpendicular to the steps and along the surface normal, respectively, as indicated in Fig. 3(a). The weighted squared difference between the measured and calculated structure factors, $\chi^{2}$, was minimized and its value used for comparison between the different models. The best fits were obtained by accounting for an atomic-scale r.m. s. roughness of $1.4 \AA^{56}$, in very good agreement with the STM measurements.

After optimization of the atomic displacements in the $\mathrm{x}$ - and $\mathrm{z}$ directions the agreement between measured and calculated structure factors, $\chi^{2}$, are $1.65,2.95$, and 2.14 for Models A, B, and $\mathrm{C}$, respectively. The final fits were obtained by fixing the $\mathrm{z}$ -

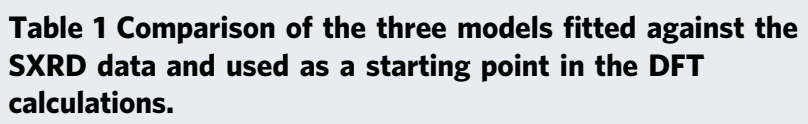

\begin{tabular}{|c|c|c|c|c|c|c|c|}
\hline & \multicolumn{4}{|c|}{$\chi^{2}$} & \multicolumn{3}{|c|}{$\Delta \sigma\left[\mathrm{meV} / \AA^{2}\right]$} \\
\hline & Unrelax. & Bare & Occup. & Addit. 0 & Bare & $\mathrm{H}_{2}$ & $\mathrm{H}_{2} \mathrm{O}$ \\
\hline Model A & 5.04 & 1.65 & 1.63 & 1.52 & 0 & -29 & -48 \\
\hline Model B & 9.76 & 2.95 & - & 3.23 & 12 & -30 & -47 \\
\hline Model C & 5.59 & 2.14 & - & 2.17 & 1 & -13 & -34 \\
\hline
\end{tabular}

The normalized goodness-of-fit, $\chi^{2}$, from the SXRD fits are given for four different cases: (Unrelax.) the unrelaxed models of the adsorbate-free surface, (bare) the adsorbate-free, bare surface after relaxation, (Occup.) with the occupancy fitted for the two top $\mathrm{Zn}-\mathrm{O}$ groups as explained in the main text, and (Addit. O) for one additional O-atom adsorbed on the surface (hydrogen atoms are not visible by $\mathrm{X}$-rays). The surface energies $(\Delta \sigma)$ from the DFT
(a) calculations are also given for three different situations: the bare surface, with dissociated $\mathrm{H}_{2}$. and with dissociated $\mathrm{H}_{2} \mathrm{O}$.

position of $\mathrm{O}$-atoms 2 and 14 and the x-position of $\mathrm{O}$-atoms 2, 5, and 9 to their bulk positions because it was found that the data are not particularly sensitive to these parameters, resulting in nonphysical bond lengths. The ranking of the models, with Model A corresponding best to the experimental surface structure and Model B the worst, is consistent with the ranking prior to fitting (see the first two columns in Table 1) and was found to hold for all the different approaches we tried. Thus, even with relatively large errors on the oxygen atomic positions the distinction between the three surface models, that differ in particularly in their surface composition with one $\mathrm{Zn}-\mathrm{O}$ pair being missing or present, is robust. The full set of CTR data obtained from the as prepared surface is shown in Fig. S6 together with the calculated structure factors for the three models. The atomic displacements for the best fit of Model A are shown in Table S1. The most significant displacements in both the $\mathrm{x}^{-}$and $\mathrm{z}^{-}$- directions are observed in the first four layers where the atoms are either undercoordinated or directly neighboring under-coordinated atoms.

Starting from the optimized Model A, also the occupancy of the $\mathrm{Zn}-\mathrm{O}$ pairs corresponding to Model $\mathrm{B}$ and $\mathrm{C}$, as indicated in Fig. 3(a), were fitted. The occupancy is fitted to be 0.98 and 1.00, respectively. An occupancy of 1 for both these $\mathrm{Zn}-\mathrm{O}$ pairs correspond to the bulk-terminated Model A. Fitting the occupancy only gave minor improvements in the $\chi^{2}$, from 1.65 for the pure Model A to 1.62 .

To summarize the SXRD results: Model A, having the lowest $\chi^{2}$ value, shows the best correspondence to the experimental surface structure. Models B and C can, therefore, already be ruled out on the grounds of the SXRD data analysis.

DFT calculations and comparisons to experiments. To further analyze our assignment of the surface structure DFT calculations were performed. The surface energies at room temperature referenced to the bare surface (structural model A) surface are shown in Table 1. Model B was found to have a considerably higher surface energy than the other two models, which have similar stability. This is consistent with the SXRD fits (also shown in Table 1) that show that Model B has the worst agreement with the experimental data.

To corroborate the assignment of the model for the surface structure, the $\mathrm{O}$ 1s CLS were calculated and compared to experiments. The CLS for Model A are shown in Fig. 4(a)-(c), whereas the results for Model B and $\mathrm{C}$ are given in Fig. S7. For all three bare surfaces we obtain a large negative CLS for O-surface atoms coordinated by only two $\mathrm{Zn}$-ions for all three bare surface models. Such a negatively shifted component is not observed in the measured O 1s XP-spectra in Fig. 2(a) and (b). 

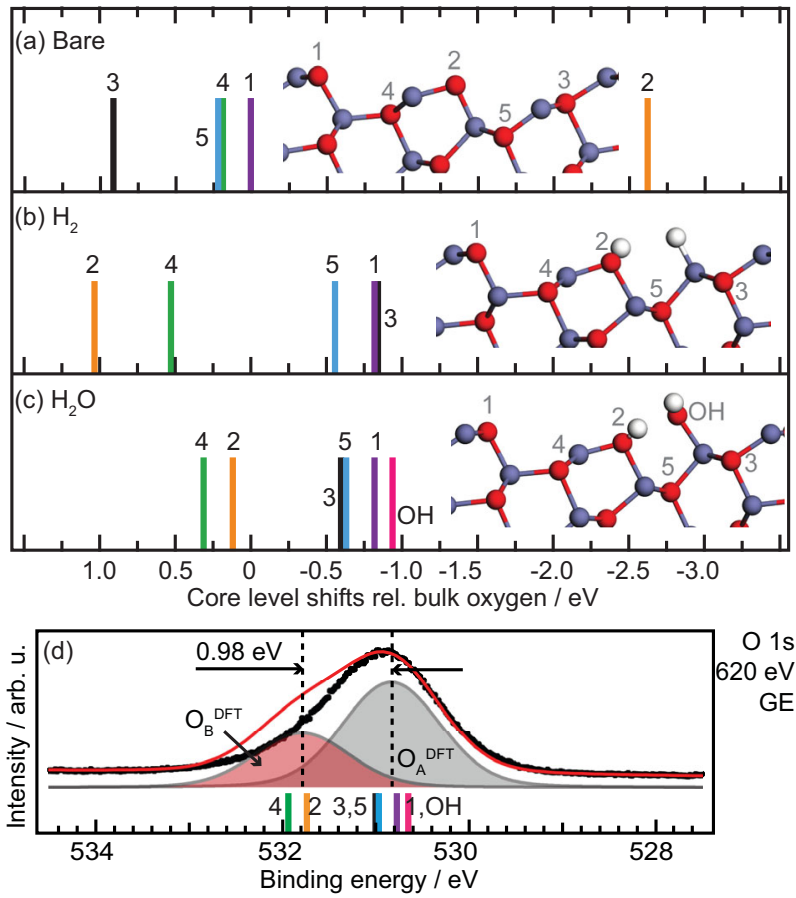

Fig. 4 Calculated $\mathbf{O}$ 1s core level shifts of Model A. a Calculated $O$ 1s core level shifts for Model A without adsorbates, b with $\mathrm{H}_{2^{-}}$, and $\mathbf{c} \mathrm{H}_{2} \mathrm{O}$ adsorbates. Numbers refer to atoms as indicated in the structural models. Atomic colorcode: $\mathrm{H}$ (white), $\mathrm{O}$ (red), $\mathrm{Zn}$ (blue). In both cases the coverage was one molecule per surface unit cell. d Simulated XP-spectra (red line) based on the CLS shown in panel c compared to the measured $\mathrm{O}$ 1s in GE (black dots). The measured data is the same as in Fig. 2(b).

Attempting to explain the absence of a peak at lower binding energies, we also performed calculations for adsorption of two common residual gases in vacuum, namely hydrogen and water. In both cases the coverage was one molecule per surface unit cell. The adsorption of both molecules has a significant influence on the position of the CLS, see Fig. 4(b) and (c). For the energetically more favorable dissociative water adsorption, the presence of $\mathrm{OH}$-groups results in the shift of O 1s CLS of the oxygen adjacent to the adsorbate (position 3 in Fig. 4), from 0.9 to $-0.6 \mathrm{eV}$ versus bulk oxygen. This shift is consistent with the formal destabilization of the adjacent $\mathrm{Zn}$ ion by the presence of the additional $\mathrm{OH}$ group. A comparable but smaller shift towards lower binding energies is also observed for the second $\mathrm{O}$ (position 5 in Fig. 4) adjacent to the destabilized $\mathrm{Zn}$ ion. The under-coordinated $\mathrm{O}$, with a relative binding energy of $-2.6 \mathrm{eV}$ for the bare surface is through water adsorption converted into an $\mathrm{OH}$ group and shifted in the opposite direction to $0.1 \mathrm{eV}$ versus $\mathrm{ZnO}$ bulk (position 2). Interestingly, an even more negative CLS of $-0.9 \mathrm{eV}$ is calculated for the second $\mathrm{OH}$-group. The very negative relative CLS of the $\mathrm{OH}$ group coordinated to only one $\mathrm{Zn}$-cation at the $\mathrm{ZnO}$ surface is different from what was observed earlier at more ionic oxides surface, such as $\mathrm{MgO}(100)$ where $\mathrm{OH}$ groups appear at a CLS of approx. $+2 \mathrm{eV}^{57}$ with respect to the bulk oxygen. The negative shift with respect to the bulk oxygen is a consequence of efficient screening of the core hole and stabilization by hydrogenbonding. A discussion about the differences between $\mathrm{ZnO}$ and $\mathrm{MgO}$ can be found in the Supplementary Information, section "Comparison to O 1s in $\mathrm{MgO}(100)$ " (Fig. S8). The positive CLS calculated for position 2 and 4 relative to the other positions after adsorption of $\mathrm{H}_{2} \mathrm{O}$ indicate that a second O-component should be present in the XP-spectra, in line with the observed $\mathrm{O}_{B}$ component. Note that the $\mathrm{O}$-atoms in position 2 and 4 are part of the $\mathrm{ZnO}$-structure, not additional $\mathrm{OH}$. The $\mathrm{OH}$-group adsorbed on the surface after $\mathrm{H}_{2} \mathrm{O}$ dissociation has a CLS that place it close to the $\mathrm{O}$-atom in position 1 . For the less favorable $\mathrm{H}_{2}$ adsorption (adsorption energy of $1.12 \mathrm{eV}$ ) the CLS are similar to the $\mathrm{H}_{2} \mathrm{O}$ case, however, with a larger spread that matches the experimental observations less well than $\mathrm{H}_{2} \mathrm{O}$ adsorption.

In Fig. 4(d) the spectrum simulated from the CLS for $1 \mathrm{H}_{2} \mathrm{O}$ on Model A is compared to the measured spectrum. The simulated spectra were obtained by introducing six components, one for each oxygen atom, with identical intensity and width, and the relative CLS fixed to the calculated values. The width of the components was fitted, as was the $\mathrm{BE}$ position relative to the measurements. After fitting of the width and relative position the six components were summarized into two groups, as presented in Fig. 4(d): $\mathrm{O}_{A}^{\mathrm{DFT}}$ composed of oxygen in position 1, 3, 5, and $\mathrm{OH}$, and $\mathrm{O}_{B}^{\mathrm{DFT}}$ composed of position 2 and 4 . The calculated spectrum is in good agreement with the one measured, aside from the higher intensity and larger shift of the $\mathrm{O}_{B}$ component in the calculated spectrum. Both these differences can be attributed to that the XPS measurements, though extremely surface sensitive, include near surface atoms (probing depth $\sim 4 \AA$ in GE) that are not taken into account in the calculated spectra. Contributions from subsurface atoms may overlap with the calculated components, we can however not resolve this in the measurements. Note also that the high surface sensitivity of the XPS measurements means that we do not observe the bulk O1s reference species with "0" core level shift.

Thus, we have concluded from the SXRD measurements that Model A agrees best with the surface structure obtained experimentally. The DFT calculations confirm that this is a stable surface structure. However, comparing with the DFT calculations we see that the XP-spectra can only be explained by the presence of one dissociated $\mathrm{H}_{2} \mathrm{O}$ molecule per surface unit cell on one $\mathrm{Zn}$ - and $\mathrm{O}$ - site. The structure for molecular and dissociate water adsorption obtained here have similarities to the ones previously reported for defected $\mathrm{ZnO}(0001)^{34,58}$ and also to other oxide surfaces, in particular with regards to the $\mathrm{OH}$ group binding in a bridge configuration between two $\mathrm{Zn}$-atoms and the $\mathrm{H}$-ion occupying an oxygen site. Surprisingly, we do not observe this $\mathrm{OH}$-species experimentally as the CLS overlaps with those of other O-sites on the surface and care needs to be taken when drawing conclusions on whether the surface is clean or not based purely on XPS measurements. The DFT calculations indicate that the adsorption and dissociation of water at the twofoldcoordinated $\mathrm{O}$ (position 2) and the adjacent under-coordinated $\mathrm{Zn}$ ion is strongly exothermic and correspondingly results in a significant stabilization. The adsorption energies are strong on both Model A and B, making them energetically similar.

Revisiting the CTR data with the dissociated water in mind an $\mathrm{O}$-atom corresponding to that of the $\mathrm{OH}$-group was added to Models A and B as these model were found to be energetically similar in the DFT calculations. For both models the addition of one O-atom lead to some adjustments of the atomic displacements particularly in the first layer, however, the changes are comparatively small as the sensitivity to O-atoms in the SXRD measurements is low relative to the $\mathrm{Zn}$-atoms. For Model A the additional $\mathrm{O}$-atom and relaxation result in a slight improvement of the $\chi^{2}$ to 1.52 (from 1.65). For Model B and C on the other hand, the addition of an O-atom lead to a worse $\chi^{2}$. See also Table 1, column 4, and further discussion in the Supplementary Information, section "SXRD fit with additional O-atom" (Fig. S9 and Table S2).

To attempt to explain why the clean bulk-terminated surface is not stable even under the best UHV conditions at room temperature and further explore the presence of adsorbates on 


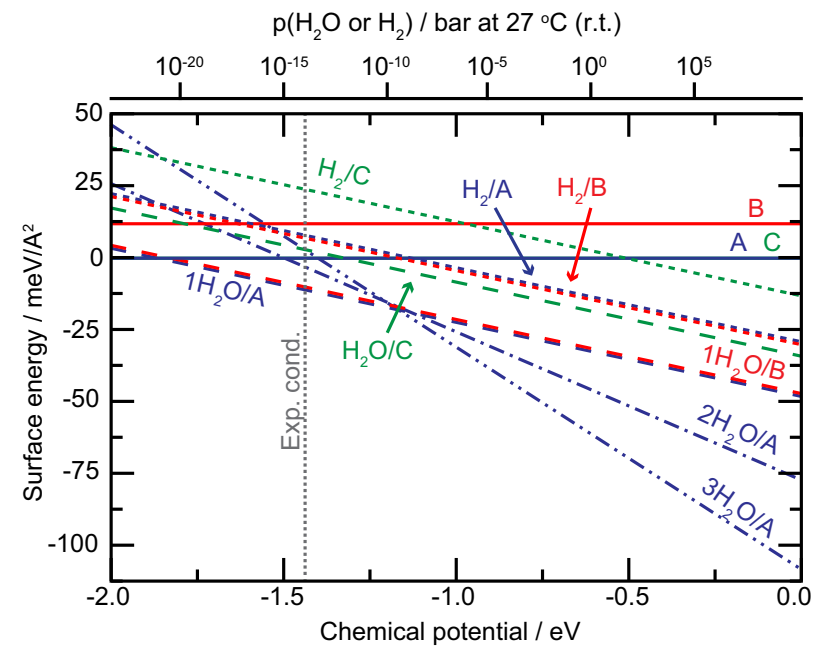

Fig. 5 Phase diagram for the bare, water, and hydrogen covered surface models. The surface stability for bare, water and hydrogen covered A (blue), B (red), and C (green) surface models are displayed. All surface models are considered for the case of a bare surface (line), one adsorbed water molecule (dashed line) and one adsorbed hydrogen molecule (dotted line). Additionally, Model A is considered with two and three water molecules (dash-dotted line and dash-dot-dotted line, respectively). The energies are reported with respect to the bare surface energy of Model A. The chemical potential corresponding to the experimental conditions during surface analysis $(-1.44 \mathrm{eV})$ is indicated by a vertical line.

the surface a phase diagram was constructed (Fig. 5) using ab intio thermodynamics ${ }^{59}$. The relative surface energies shown in Table 1 are taken at the line indicating the experimental conditions. As mentioned above, the bare Model B shows considerably higher surface energy than the other two models, which exhibit similar stability. For all three surfaces dissociative adsorption of one water molecule is favored over one hydrogen molecule. Focusing on the energetically more favorable water adsorption we find that the adsorption energy of one water molecule is $1.85,2.30$, and $1.35 \mathrm{eV}$ on Model A, B, and C, respectively. The high adsorption energy is related to the undercoordinated oxygen atom, yielding dissociative adsorption with a hydrogen bond between the two $\mathrm{OH}$-groups, see Fig. 3(b). The calculated atomic displacements are shown in Table S3. For Model A, we also investigated the possibility with two and three water molecules and the adsorption energy per water molecule is calculated to be 1.50 and $1.40 \mathrm{eV}$, respectively (see Fig. S10 for structures). For Model A, the situation with one adsorbed (dissociated) molecule is preferred between water chemical potentials of $-1.85 \mathrm{eV}$ and $-1.15 \mathrm{eV}$. At higher chemical potentials three molecules are preferred. We note that the thermodynamically stable phase during the experimental conditions is Model A with one adsorbed water molecule, in agreement with the previous conclusions.

Thus, we have shown that the $\mathrm{ZnO}(10 \overline{1} 4)$ is a long-range ordered vicinal oxide surface. Using SXRD, XPS, STM, and DFT, we determine that the surface has a bulk truncated structure with $\mathrm{OH}$ present on two uncoordinated sites under all our experimental conditions. DFT shows that this dissociated $\mathrm{H}_{2} \mathrm{O}$ further stabilizes the surface significantly without altering the surface structure. The surface remains highly active for water adsorption and dissociation through the two remaining under-coordinated $\mathrm{Zn}$-atoms also when it is partially covered by $\mathrm{OH}$-groups. The experimentally observed core level shifts for the as prepared surface can consistently be explained by DFT. Such a surface with a high density of under-coordinated step sites is an ideal playground for future studies of how these sites influence the catalytic properties of $\mathrm{ZnO}$.

\section{Methods}

Sample preparation. The $\mathrm{ZnO}(10 \overline{1} 4)$ surface was cleaned through cycles of sputtering $\left(500 \mathrm{eV} \mathrm{Ar}{ }^{+}, 20 \mathrm{~min}\right.$, room temperature (r.t.)) and subsequent annealing at $650^{\circ} \mathrm{C}$ (residual pressure $\mathrm{p}_{R}<3 \cdot 10^{-9} \mathrm{mbar}$ ). To determine the optimal annealing temperature the terrace size was studied as a function of the annealing temperature and time by STM (see Supplementary Methods, Fig. S1(a)). The surface order and the concentration of defects was not seen to improve by annealing to temperatures over $650^{\circ} \mathrm{C}$ or longer than $10 \mathrm{~min}$. The terrace height is always $\sim 1.2 \AA$. Exampe STM images taken after annealing to $630,670^{\circ} \mathrm{C}$ and after $\mathrm{O}_{2}$ annealing at $670{ }^{\circ} \mathrm{C}$ are shown in Fig. S1(b)-(d).

Scanning tunneling microscopy (STM). The STM measurements were performed in a Scienta Omicron VT SPM situated at the DESY NanoLab ${ }^{60}$. All measurements were performed at room temperature in constant current mode using etched W-tips (Ar-sputtered and annealed after introduction to UHV). The residual pressure in the chamber was $\mathrm{p}_{R}<5 \cdot 10^{-11}$ mbar. The temperature was measured by a thermocouple close to the sample, which was cross-calibrated at an earlier time with a thermocouple spot-welded to the side of a metal crystal mounted on the same type of plate as used for the $\mathrm{ZnO}$ sample. The images were post processed using Gwyddion ${ }^{61}$.

X-ray photoelectron spectroscopy (XPS). XPS measurements were carried out at the former $i 311$ beamline at the MAX IV Laboratory $\left(\mathrm{p}_{R}<1 \cdot 10^{-10} \mathrm{mbar}\right)^{62}$. No contaminants were observed by XPS on the surface after cleaning (see Fig. S11). O $1 \mathrm{~s}$ spectra were collected in both normal- (NE) and grazing emission (GE) at $60^{\circ}$ from the normal with $620 \mathrm{eV}$ photon energy with a total energy resolution of 130 $\mathrm{meV}$. The O 1s XP-spectra were curve fitted using convoluted Doniach-Šunjić and Gaussian functions with a linear background and no asymmetry. During fitting the Lorentzian full width at half maximum for all components was fixed to $0.18 \mathrm{eV}$, in accordance with ref. ${ }^{63}$. Further, the Gaussian full width at half maximum (GFWHM) of the $\mathrm{O}_{A}$ and $\mathrm{O}_{B}$ components were restricted to be the same. The GFWHM for $\mathrm{O}_{A}$ and $\mathrm{O}_{B}$ was found to be $1.1 \mathrm{eV}$, while $\mathrm{O}_{C}$ has a GFWHM of 1.3 $\mathrm{eV}$. The binding energy position of the $\mathrm{O}_{B}$ component was determined through difference spectra between the normal and grazing measurements.

Surface X-ray diffraction (SXRD). The SXRD measurements were performed on the surface diffraction beamline ID03 at the European Synchrotron Radiation Facility (ESRF) ${ }^{64}$, using the dedicated UHV SXRD chamber $\left(\mathrm{p}_{R}<1 \cdot 10^{-10} \mathrm{mbar}\right)$ and a photon energy of $18 \mathrm{keV}$. The SXRD data was analyzed using ROD from the ANAROD package ${ }^{56}$. During the structural refinement the atoms in the first seven layers along the surface normal were allowed to vary. Displacements in deeper layers have no or a very minor impact on the quality of the fit and were, therefore, kept fixed to their bulk values. During the fitting the Debye-Waller factors of all atoms were kept fixed to their bulk values of 0.651 and $0.64 \AA^{2}$ for the $\mathrm{Zn}$ - resp. Oatoms $s^{65,66}$. Fitting or manually modifying the factors had no significant influence on the $\chi^{2}$ values. See Supplementary Information, section "SXRD models and fits" for further details.

DFT calculations. Density functional theory (DFT) calculations were performed with the Vienna ab initio simulation package (VASP; version 5.4.1) using the $\mathrm{PBE}^{67}$ functional with a Hubbard $\mathrm{U}$ of $7.5 \mathrm{eV}$ for the $\mathrm{Zn} 3 \mathrm{~d}$ orbitals. The core electrons were treated within the projector augmented wavefunction (PAW) method $^{68}$ and the Kohn-Sham orbitals were expanded in a plane wave basis, truncated at $550 \mathrm{eV}$. Properties of the $\mathrm{ZnO}(10 \overline{1} 4)$ surface were obtained using a 13 monolayers thick slab where the bottom 9 monolayers were fixed to the bulk positions. slab of this thickness was required to obtain converged surface-bulk core level shifts. The calculations were done using a $(1 \times 1)$ surface unit cell together with a $1 \times 5 \times 1 \mathrm{k}$-point set. All structures were relaxed using the conjugate gradient algorithm implemented in VASP. The obtained lattice parameters of $a=b=$ $3.19 \AA$ and $c=5.12 \AA$ are in good agreement with experiments ${ }^{69}$. Core level shifts were calculated with final state effects assuming complete screening of the core hole. In this method, the CLS is calculated as the total energy difference between the atom at consideration and a reference atom, which in our caseis a bulk atom. The core level shifts were calculated using a $(1 \times 3)$ surface cell with one k-point. The systems with a core hole were constructed by generating a PAW potential with an electron hole in the 1s shell of O. Charge neutrality was maintained in the calculations of the core level shifts by adding a jellium background ${ }^{70}$.

\section{Data availability}

Data not shown can be found in the Supplementary Information, the Supplementary Data files (Supplementary Data 1-4: structures used for the SXRD fits, Supplementary Data 5: measured and calculated structure factors for the best fitting model) or can be requested from the corresponding author. 
Received: 4 March 2020; Accepted: 4 December 2020;

Published online: 20 January 2021

\section{References}

1. Wöll, C. The chemistry and physics of zinc oxide surfaces. Prog. Surf. Sci. 82, 55-120 (2007).

2. Ellmer, K., Klein, A., \& Rech B. (eds) Transparent Conductive Zinc Oxide: Basics and Applications in Thin Film Solar Cells. (Springer Berlin Heidelberg, 2007).

3. Schmidt-Mende, L. \& MacManus-Driscoll, J. L. ZnO-nanostructures, defects, and devices. Mater. Today 10, 40 (2007).

4. Mugarza, A. \& Ortega, J. E. Electronic states at vicinal surfaces. J. Phys, Condens. Matter 15, S3281-S3310 (2003).

5. Farber, R. G. et al. Exposure of $\operatorname{Pt}\left(\begin{array}{lll}5 & 5 & 3\end{array}\right)$ and $\mathrm{Rh}\left(\begin{array}{lll}1 & 1 & 1\end{array}\right)$ to atomic and molecular oxygen: do defects enhance subsurface oxygen formation? J. Phys.: Condens. Matter 29, 164002 (2017).

6. Badan, C., Koper, M. T. M. \& Juurlink, L. How well does Pt(211) represent Pt $[\mathrm{n}(111) \times(100)]$ surfaces in adsorption/desorption? J. Phys. Chem. C 119, 13551-13560 (2015).

7. Walter, A. L. et al. X-ray photoemission analysis of clean and carbon monoxide-chemisorbed platinum(111) stepped surfaces using a curved crystal. Nat. Commun. 6, 8903 (2015).

8. Pham-Van, L., Kurnosikov, O. \& Cousty, J. Evolution of steps on vicinal (0001) surfaces of a-alumina. Surf. Sci. 411, 263-271 (1998).

9. Kurnosikov, O., Van, L. P. \& Cousty, J. High-temperature transformation of vicinal (0001) Al2O3-a surfaces: an AFM study. Surf. Interf. Anal. 29, 608-613 (2000).

10. Nguyen, T., Bonamy, D., Van, L. P., Barbier, L. \& Cousty, J. Coarsening of two-dimensional $\mathrm{Al}_{2} \mathrm{O}_{3}$ islands on vicinal $(1,-1,0,2)$ sapphire surfaces during annealing in air. Surf. Sci. 602, 3232-3238 (2008).

11. Thune, E. et al. Nanostructured sapphire vicinal surfaces as templates for the growth of self-organized oxide nanostructures. Appl. Surf. Sci. 256, 924-928 (2009).

12. Verre, R. et al. Equilibrium faceting formation in vicinal $\mathrm{Al}_{2} \mathrm{O}_{3}$ (0001) surface caused by annealing. Surf. Sci. 606, 1815-1820 (2012).

13. Torrelles, X. et al. Atomic structure of a long-range ordered vicinal surface of SrTiO3. Surf. Sci. 589, 184-191 (2005).

14. Joumard, I. et al. Nanostructure of a vicinal surface of SrTiO3: Order on two length scales. Phys. Rev. B 74, 205411 (2006).

15. Theis, C. D., Yeh, J., Schlom, D. G., Hawley, M. E. \& Brown, G. W. The influence of vicinal $\mathrm{SrTiO} 3$ surfaces on the growth and ferroelectric properties of epitaxial Bi4Ti3O12 thin films. Mater. Sci. Eng. B 56, 228-233 (1998).

16. Dekkers, J. M. et al. Monocrystalline $\mathrm{YBa} 2 \mathrm{Cu} 3 \mathrm{O} 7-\mathrm{x}$ thin films on vicinal SrTiO3(001) substrates. Appl. Phys. Lett. 83, 5199-5201 (2003).

17. Sanchez, F. et al. Giant step bunching in epitaxial SrRuO3 films on vicinal SrTiO3(001). Thin Solid Films 495, 159-164 (2006).

18. Miccio, L. A. et al. Interplay between steps and oxygen vacancies on curved TiO2(110). Nano Lett. 16, 2017-2022 (2016).

19. Rieboldt, F., Bechstein, R., Besenbacher, F. \& Wendt, S. Vicinal rutile TiO2 surfaces and their interactions with O2. J. Phys. Chem. C 118, 3620-3628 (2014).

20. Deng, X., Sorescu, D. C. \& Lee, J. Methanol oxidation to formaldehyde promoted at the step sites of ultrathin ZnO. Top Catal. 61, 499-508 (2018).

21. Diebold, U., Li, S.-C. \& Schmid, M. Oxide surface science. Ann. Rev. Phys. Chem. 61, 129-148 (2010).

22. Akhter, S., Lui, K. \& Kung, H. H. Comparison of the chemical propertles of the zinc-polar, the oxygen-polar, and the nonpolar surfaces of ZnO. J. Phys. Chem. 89, 1958-1964 (1985).

23. Cheng, W. H. \& Kung, H. H. Chemical properties of anion vacancies on zinc oxide. Surf. Sci. Lett. 102, L21-L28 (1981).

24. Dulub, O., Boatner, L. A. \& Diebold, U. STM study of the geometric and electronic structure of $\mathrm{ZnO}(0001)-\mathrm{Zn},(000-1)-\mathrm{O}$, (10-10), and (11-20) surfaces. Surf. Sci. 519, 201-207 (2002).

25. Lindsay, R. et al. Impact of defects on the surface chemistry of $\mathrm{ZnO}(000-1)-\mathrm{O}$. J. Am. Chem. Soc. 124, 7117-7122 (2002).

26. Kresse, G., Dulub, O. \& Diebold, U. Competing stabilization mechanism for the polar $\mathrm{ZnO}(0001)-\mathrm{Zn}$ surface. Phys. Rev. B 68, 245409 (2003).

27. Meyer, B. First-principles study of the polar o-terminated $\mathrm{ZnO}$ surface in thermodynamic equilibrium with oxygen and hydrogen. Phys. Rev. B 69, 045416 (2004)

28. Meyer, B. et al. Partial dissociation of water leads to stable superstructures on the surface of zinc oxide. Angew. Chem. 43, 6641-6645 (2004).

29. Ketteler, G. \& Ranke, W. Epitaxial iron oxide growth on a stepped Pt(9 11 11) surface. J. Vac. Sci. Technol. 23, 1055-1060 (2005).
30. Valtiner, M., Borodin, S. \& Grundmeier, G. Preparation and characterisation of hydroxide stabilised $\mathrm{ZnO}(0001)-\mathrm{zn}-\mathrm{OH}$ surfaces. Phys. Chem. Chem. Phys. 9, 2406-2412 (2007).

31. Ostendorf, F., Torbrügge, S. \& Reichling, M. Atomic scale evidence for faceting stabilization of a polar oxide surface. Phys. Rev. B 77, 041405(R) (2008).

32. Valtiner, M., Todorova, M., Grundmeier, G. \& Neugebauer, J. Temperature stabilized surface reconstructions at polar $\mathrm{ZnO}(0001)$. Phys. Rev. Lett. 103, 065502 (2009).

33. Torbrügge, S., Ostendorf, F. \& Reichling, M. Stabilization of zinc-terminated $\mathrm{ZnO}(0001)$ by a modified surface stoichiometry. J. Phys. Chem. C 113, 4909-4919 (2009).

34. Valtiner, M., Todorova, M. \& Neugebauer, J. Hydrogen adsorption on polar $\mathrm{ZnO}(0001)$-zn: Extending equilibrium surface phase diagrams to kinetically stabilized structures. Phys. Rev. B 82, 165418 (2010).

35. Lauritsen, J. V. et al. Stabilization principles for polar surfaces of $\mathrm{ZnO}$. ACS Nano 5, 5987-5994 (2011).

36. Wahl, R., Lauritsen, J. V., Besenbacher, F. \& Kresse, G. Stabilization mechanism for the polar $\mathrm{ZnO}(000-1)$-o surface. Phys. Rev. B 87, 085313 (2013).

37. Mora-Fonz, D. et al. Why are polar surfaces of $\mathrm{ZnO}$ stable? Chem. Mater. 29 , 5306-5320 (2017).

38. Hellström, M., Beinik, I., Broqvist, P., Lauritsen, J. V. \& Hermansson, K. Subsurface hydrogen bonds at the polar zn-terminated zno(0001) surface. Phys. Rev. B 94, 245433 (2016).

39. Niederhausen, J. et al. X-ray standing waves reveal lack of $\mathrm{OH}$ termination at hydroxylated $\mathrm{ZnO}(0001)$ surfaces. Phys. Rev. Mater. 4, 020602 (2020).

40. Zheng, H., Gruyters, M., Pehlke, E. \& Berndt, R. Magic vicinical zinc oxide surfaces. Phys. Rev. Lett. 111, 086101 (2013).

41. Chan, K. S. et al. Equilibrium shape of nano-cavities in $\mathrm{H}$ implanted $\mathrm{ZnO}$. Appl. Phys. Lett. 106, 212102 (2015).

42. Grånäs, E., Arndt, B., Seitz, C., Wagstaffe, M. \& Stierle, A. Atomic scale step structure and orientation of a curved surface zno single crystal. J. Phys. Chem. 152, $074705(2020)$

43. Wang, $\mathrm{H}$. et al. Effects of nitrogen dopants on the atomic step kinetics and electronic structures of O-polar ZnO. Nanoscale 8, 4381 (2016).

44. Tasker, P. W. The stability of ionic crystal surfaces. J. Phys. C 12, 4977-4984 (1979).

45. von Hoegen, M. H. Growth of semiconductor layers studied by spot profile analysing low energy electron diffraction-part II1. Zeitschrift für Kristallographie 214, 591-629 (1999).

46. Roman Kováčik, D. M. \& Meyer, B. F centers versus dimer vacancies on zno surfaces: characterization by stm and sts calculations. Angew. Chem. 46, 4894 (2007).

47. Qiu, H., Meyer, B., Wang, Y. \& Wöll, C. Ionization energies of shallow donor states in $\mathrm{ZnO}$ created by reversible formation and depletion of $\mathrm{H}$ interstitials. Phys. Rev. Lett. 101, 236401 (2008).

48. Enevoldsen, G. H. et al. Imaging of the hydrogen subsurface site in $\mathrm{RutileTiO}_{2}$ Phys. Rev. Lett. 102, 136103 (2009).

49. Noei, H., Qiu, H., Wang, Y., Muhler, M. \& Wöll, C. Hydrogen loading of oxide powder particles: a transmission IR study for the case of zinc oxide. ChemPhysChem 11, 3604-3607 (2010).

50. Kunat, M., Girol, S. G., Burghaus, U. \& Woell, C. The interaction of water with the oxygen-terminated, polar surface of ZnO. J. Phys. Chem. B 107, 14350-14356 (2003).

51. Schiek, M., Al-Shamery, K., Kunat, M., Traeger, F. \& Woell, C. Water adsorption on the hydroxylated $\mathrm{H}-(1 \mathrm{x} 1) \mathrm{O}-\mathrm{ZnO}(000-1)$ surface. Phys. Chem. Chem. Phys. 8, 1505-1512 (2006)

52. Önsten, A. et al. Water adsorption on $\mathrm{ZnO}(0001)$ : transition from triangular surface structures to a disordered hydroxyl terminated phase. J. Phys. Chem. 114, 11157-11161 (2010)

53. Newberg, J. T. et al. $\mathrm{ZnO}(10-10)$ surface hydroxylation under ambient water vapor. J. Phys. Chem. B 122, 472-478 (2017).

54. Deinert, J.-C., Hofmann, O. T., Meyer, M., Rinke, P. \& Stahler, J. Local aspects of hydrogen-induced metallization of the $\mathrm{ZnO}(10-10)$ surface. Phys. Rev. B 91 , 235313 (2015)

55. Ozawa, K. \& Mase, K. Metallization of $\mathrm{ZnO}(10-10)$ by adsorption of hydrogen, methanol, and water: Angle-resolved photoelectron spectroscopy. Phys. Rev. B 81, 205322 (2010).

56. Vlieg, E. ROD: a program for surface x-ray crystallography. J. Appl. Crystallogr. 33, 401-405 (2000).

57. Paz-Borbón, L. O., Hellman, A. \& Grönbeck, H. Simulated photoemission spectra of hydroxylated $\mathrm{MgO}(100)$ at elevated temperatures. J. Phys. Chem. C 116, 3545 (2012).

58. Iachella, M. et al. Water dissociation and further hydroxylation of perfect and defective polar ZnO model surfaces. J. Phys. Chem. C 122, 21861-21873 (2018).

59. Reuter, K. \& Scheffler, M. Composition, structure, and stability of $\mathrm{RuO}_{2}(110)$ as a function of oxygen pressure. Phys. Rev. B 65, 3 (2001). 
60. Stierle, A., Keller, T. F., Noei, H., Vonk, V. \& Roehlsberger, R. DESY NanoLab. J. Large-Scale Res. Facilit. 2, A76 (2016).

61. Necas, D. \& Klapetek, P. Gwyddion: an open-source software for SPM data analysis. Cent. Eur. J. Phys. 10, 181-188 (2012).

62. Nyholm, R., Andersen, J. N., Johansson, U., Jensen, B. N. \& Lindau, I. Beamline I311 at MAX-LAB: a VUV/soft X-ray undulator beamline for high resolution electron spectroscopy. Nucl. Instrum. Methods Phys. Res. A 467-468, 520-524 (2001).

63. Campbell, J. L. \& Papp, T. Widths of the atomic K-N7 levels. At. Data Nucl. Data Tables 77, 1-56 (2001).

64. Ferrer, S. \& Comin, F. Surface diffraction beamline at ESRF. Rev. Sci. Instrum. 66, 1674-1676 (1995).

65. Sawada, H., Wang, R. \& Sleight, A. W. An electron density residual study of zinc oxide. J. Solid State Chem. 122, 148-150 (1996).

66. Schlepütz, C. M. et al. The presence of a $(1 \times 1)$ oxygen overlayer on $\mathrm{ZnO}$ (0001) surfaces and at schottky interfaces. J. Phys.: Condens. Matter 24 095007 (2012).

67. Perdew, J. P., Burke, K. \& Ernzerhof, M. Generalized gradient approximation made simple. Phys. Rev. Lett. 77, 3865-3868 (1996).

68. Blöchl, P. E. Projector augmented-wave method. Phys. Rev. B 50, 17953-17979 (1994).

69. Erhart, P., Albe, K. \& Klein, A. First-principles study of intrinsic point defects in zno: Role of band structure, volume relaxation, and finite-size effects. Phys. Rev. B 73, 205203 (2006).

70. Van Den Bossche, M. et al. Effects of non-local exchange on core level shifts for gas-phase and adsorbed molecules. J. Chem. Phys. 141, 034706 (2014).

\section{Acknowledgements}

The staff of the I311 beamline at the MAX IV Laboratory and the ID03 beamline at ESRF is gratefully acknowledged for their support during beamtimes. H.G., M.B., and A. Stierle acknowledge the support from the Röntgen-Ångström project HEXCHEM. The calculations were performed on resources provided by the Swedish National Infrastructure for Computing (SNIC).

\section{Author contributions}

E.G., B.A., M.C., G.D.L.S., and V.V. performed the SXRD measurements. E.G., J.G., and

A. Schaefer performed the XPS measurements. E.G. also performed the STM and LEED measurements and the analysis for all measurements. M.B. and H.G. performed the DFT calculations. E.G. wrote the article together with V.V., H.G., and A. Stierle All authors discussed the results and commented on the manuscript.

\section{Funding}

Open Access funding enabled and organized by Projekt DEAL.

\section{Competing interests}

The authors declare no competing interests.

\section{Additional information}

Supplementary information is available for this paper at https://doi.org/10.1038/s42004 020-00442-6.

Correspondence and requests for materials should be addressed to E.G. or A.S.

Reprints and permission information is available at http://www.nature.com/reprints

Publisher's note Springer Nature remains neutral with regard to jurisdictional claims in published maps and institutional affiliations.

\section{(c) (i)}

Open Access This article is licensed under a Creative Commons Attribution 4.0 International License, which permits use, sharing, adaptation, distribution and reproduction in any medium or format, as long as you give appropriate credit to the original author(s) and the source, provide a link to the Creative Commons license, and indicate if changes were made. The images or other third party material in this article are included in the article's Creative Commons license, unless indicated otherwise in a credit line to the material. If material is not included in the article's Creative Commons license and your intended use is not permitted by statutory regulation or exceeds the permitted use, you will need to obtain permission directly from the copyright holder. To view a copy of this license, visit http://creativecommons.org/ licenses/by/4.0/.

(C) The Author(s) 2021 